\title{
On Gradient Simplex Methods for Linear Programs
}

R. R. VEMUGANTI

rvemuganti@ubalt.edu

Merrick School of Business, University of Baltimore, MD 21201, USA

\begin{abstract}
A variety of pivot column selection rules based upon the gradient criteria (including the steepest edge) have been explored to improve the efficiency of the primal simplex method. Simplex-like algorithms have been proposed imbedding the gradient direction $(G D)$ which includes all variables whose increase or decrease leads to an improvement in the objective function. Recently a frame work has been developed in the simplex method to incorporate the reduced-gradient direction (RGD) consisting of only variables whose increase leads to an improvement in the objective function. In this paper, the results are extended to embed GD in the simplex method based on the concept of combining directions. Also mathematical properties related to combining directions as well as deleting a variable from all basic directions are presented.
\end{abstract}

Keywords: Gradient Directions, Linear Programming, Simplex Algorithm.

\section{Introduction}

Consider the linear program $(\mathrm{LP}(1))$ :

$$
\begin{array}{rc}
\operatorname{Max} & z=c^{t} x \\
\text { s.t. } & A x=b \text { and } x \geq 0
\end{array}
$$

where $c^{t}=\left(c_{1}, \ldots, c_{n}\right), b^{t}=\left(b_{1}, \ldots, b_{m}\right), x^{t}=\left(x_{1}, \ldots, x_{n}\right)$ and $A=$ $\left(a_{i j}\right)$ is a $m \times n$ matrix. Suppose $\mathrm{B}$ is the basis matrix corresponding to a feasible extreme point and let $a_{j}$ be the column corresponding to the variable $x_{j}$. Also let $x_{B}$ and $x_{N}$ be the set of basic and nonbasic variables corresponding to the basis matrix B. Partitioning the matrix A and the vector $c^{t}$ corresponding to the basic and nonbasic variables into $\mathrm{A}=(\mathrm{B}$, $\mathrm{N})$ and $c^{t}=\left(c_{B}^{t}, c_{N}^{t}\right)$, the linear programming in (1) can be written as

$$
\begin{aligned}
\operatorname{Max} & =c_{B}^{t} B^{-1} b+\bar{c}_{N}^{t} x_{N} \\
\text { s.t. } \quad x_{B}+B^{-1} N x_{N} & =B^{-1} b=\bar{b} \text { and }\left(x_{B}, x_{N}\right) \geq 0
\end{aligned}
$$

where $\bar{c}_{N}^{t}=\left(c_{N}^{t}-c_{B}^{t} B^{-1} N\right)$. Clearly the solution is optimal if $\bar{c}_{N} \leq 0$. When one or more $\bar{c}_{j}, j \in x_{N}$ are positive the objective function value may

$\dagger$ Requests for reprints should be sent to R. R. Vemuganti, Merrick School of Business, University of Baltimore, 1420 North Charles Street, Baltimore, MD 21201, USA. 
be improved by replacing one of the basic variables (pivot row) with one of the nonbasic variables $x_{j}$ (pivot column) for which $\bar{c}_{j}>0$. In the steepest unit ascent method (Dantzig(63)), the variable with the largest positive $\bar{c}_{j}$ is selected to enter the basis. To maintain primal feasibility the departing variable $x_{p}$ is chosen using the rule

$$
\bar{b}_{p} / \bar{a}_{p k}=\min _{i}\left(\bar{b}_{i} / \bar{a}_{i k}, \bar{a}_{i k}>0\right)
$$

where $\mathrm{k}$ is the entering variable and $\bar{a}_{j}=\left(\bar{a}_{i j}\right)$ is the column corresponding to $x_{j}$ in the formulation (2). If no such departing variable exists, the problem is unbounded. When there is a tie, Bland's (77) rule may be used to avoid cycling. An improvement over the unit ascent rule is to select the variable $\mathrm{k}$ for which the change in the objective function given by $\bar{c}_{k}\left(\bar{b}_{p} / \bar{a}_{p k}\right)$ is a maximum.

Computationally more expensive gradient criteria for the selection of the pivot column can be found in Abel (87), Goldfarb and Reid (77), Harris (73), and Zoutenddijk (76). For example if the $N_{p}$-norm of $\left(\bar{a}_{j}, h\right)$ is given by

$$
\begin{aligned}
N_{p}\left(\bar{a}_{j}, h\right) & =\left[\sum_{i=1}^{m}\left(\left|\bar{a}_{i j}\right|+|h|\right)^{p}\right] \quad \text { for } 1 \leq p<\infty \\
N_{\infty}\left(\bar{a}_{j}, h\right) & =\operatorname{Max}\left(\left|\bar{a}_{i 1}\right|, \ldots,\left|\bar{a}_{i n}\right|,|h|\right)
\end{aligned}
$$

then the gradient of the variable $\mathrm{j}$ is $g_{j}^{p}=\bar{c}_{j} / N_{p}\left(\bar{a}_{j}, h\right)$. Including only the positive values or negative values or both of $\bar{a}_{j}$ in the $N_{p}$ - norm and setting $\mathrm{h}=1$ or $\bar{c}_{j}$, a variety of gradients can be generated. Under the gradient criteria, the variable with the largest gradient value for which $\bar{c}_{j}>0$ is selected. The gradient $g_{j}^{2}$ when $\mathrm{h}=1$ corresponds to the steepest edge. Abel (87) reports the gradient criterion for $\mathrm{p}=1$ and $\mathrm{h}=1$, appears to perform better requiring fewer iterations when compared with several other gradients by changing the values of $\mathrm{p}$. Computational experiments by Goldfarb and Reid (77) indicate that the steepest edge criterion is more efficient than the rule of selecting the variable with the most positive $\bar{c}_{j}$. Attempts to bring more than one variable into the basis simultaneously are limited to two variables (Paranjape (65) ) due to excessive computational effort. Another approach called gradient method, which attempts to combine all eligible variables into a bundle is addressed by Beilby (76), Eislet and Sandblom (90), Fathi and Murty (89), Graves and Wolfe (64), Heady and Candler (58), Kallio and Porteus (78), Mitra, Tamiz and Yadegar (88). Following the development of Kallio and Porteus (78), suppose $\mathrm{x}$ is a feasible solution corresponding to a basis where some nonbasic variables are permitted to be at a positive level. For each nonbasic variable $\mathrm{j}$, 
let $d_{j}=\left(d_{i j}\right)$, be an $\mathrm{n}$ dimensional vector given by

$$
d_{i j}= \begin{cases}\bar{a}_{i j} & \text { if } i \text { is a basic variable } \\ 1 & \text { if } i=j \\ 0 & \text { otherwise. }\end{cases}
$$

Determine the direction d by combining the columns $d_{j}$, with weights given by

$$
w_{j}= \begin{cases}\bar{c}_{j} & \text { if } \bar{c}_{j}<0 \text { and } x_{j}>0 \text { or } \bar{c}_{j}>0 \\ 0 & \text { otherwise }\end{cases}
$$

Now consider the new solution $\bar{x}=x+\alpha d$ where $d=\sum_{j=1}^{n} w_{j} d_{j}$. Find the largest step size $\alpha^{*}$ for which $\bar{x} \geq 0$. Clearly the new solution is given by $\bar{x}=x+\alpha^{*} d$ and the increase in the objective function is $\alpha^{*} \sum_{j=1}^{n} \bar{c}_{j}^{2}$. When a nonbasic variable decreases to zero in the solution, the procedure is continued. When one or more basic variables are driven to zero, one of these basic variables is replaced by the first candidate in the list of the nonbasic variables. The departing variable is added to the list of the nonbasic variables. Fathi and Murty (89) and Mitra, Tamiz and Yadegar (88), provide a mechanism to construct a basic feasible solution from $\bar{x}$ with the objective function value $\geq c^{t} \bar{x}$ before generating the next direction. This method is proven to converge (Kallio and Porteus (78)) in a finite number of iterations when the LP is non degenerate. As noted by Graves and Wolfe (64), this approach is similar to the gradient methods (Rosen (61) and Kreko (68)), in using the gradient to determine the desired direction of movement and is not conveniently illustrated geometrically.

Eislet and Sandblom (90) and Zipkin (80), developed a framework to embed reduced-gradient direction (RGD) called external pivoting in the simplex method where the variables in the gradient direction are restricted only to those nonbasic variables for which $\bar{c}_{j}>0$. In this paper the results are extended to develop a frame work to incorporate the gradient direction (GD) in the simplex method and two algorithms are presented to implement the scheme. The next section deals with the frame work to embed RGD and GD in the simplex method.

\section{Gradient Simplex Method Framework}

Suppose $Q_{k}$ is a subset of variables $\mathrm{v}=(1,2, \ldots, \mathrm{n})$. Define a variable $y_{k}$ with a column vector $d_{k}$ and the coefficient in the objective function $f_{k}$ 
given by

$$
d_{k}=\sum_{j \in Q_{k}} w_{j k} a_{j} \text { and } f_{k}=\sum_{j \in Q_{k}} w_{j k} c_{j}
$$

where $\left|w_{j k}\right|>0$ for $j \in Q_{k}$ and $w_{j k}=0$ otherwise and $\sum_{j \in Q_{k}}\left|w_{j k}\right|=1$. Excluding the singleton sets and the empty set, noting the fact that each weight $w_{j k}$ can be positive or zero or negative, the number of y-variables one can construct is $r=3^{n}-(n+1)$. Now consider the LP (3) with $n$ $\mathrm{x}$-variables and $\mathrm{r} \mathrm{y}$-variables given by

$$
\begin{array}{cc}
M a x & z=c^{t} x+f^{t} y \\
\text { s.t. } & A x+D y=b, \quad I x+W y \geq 0 \quad \text { and } x \geq 0, y \geq 0
\end{array}
$$

where $D=\left(d_{1}, \ldots, d_{r}\right)$ is a $m \times r$ matrix, $W=\left(w_{j k}\right)$ is a $n \times r$ matrix, $I$ is a $n \times n$ identity matrix and $y^{t}=\left(y_{1}, \ldots, y_{r}\right)$. It is easy to verify that if $x^{*}$ is a feasible solution to $\operatorname{LP}(1)$ then $\mathrm{x}=x^{*}$ and $\mathrm{y}=0$ is a feasible solution to $\operatorname{LP}(3)$. Conversely if $\left(x^{*}, y^{*}\right)$ is a feasible solution to $\operatorname{LP}(3)$ then $x=x^{*}+W y^{*}$ is a feasible solution to $\operatorname{LP}(1)$. Since the corresponding objective functions are equal it is clear that an optimal solution to $\mathrm{LP}(1)$ can be found by solving $\mathrm{LP}(3)$.

When the weights $w_{i j}$ are restricted to be nonnegative (RGD), the number of $\mathrm{y}$-variables that can be formed is reduced to $r=2^{n}-(n+1)$ and the second set of constraints in formulation 4 is redundant. As described in Eislet and Sandblom (90) and Zipkin(80), to embed the RGD in the simplex method, let $\bar{a}_{i j}, \bar{b}_{i}$ and $\bar{c}_{j}$ be the elements of any tableau. Construct the variable $y_{k}$ with weights

$$
w_{j k}= \begin{cases}\bar{c}_{j} / \gamma & \text { if } j \in Q_{k}=\left(j \mid \bar{c}_{j}>0\right) \\ 0 & \text { otherwise }\end{cases}
$$

where $\gamma=\sum_{j \in Q_{k}} \bar{c}_{j}$. Determine the corresponding column vector $d_{k}$ and the objective function coefficient $f_{k}$ from (3). Pivot the variable $y_{k}$ into the basis and continue the procedure till an optimal solution is found. In the next section the results are extended to embed GD in the simplex method based on the concept of combining directions and related mathematical properties.

\section{Gradient Simplex Method}

It appears that the formulation (4) requires a basis size of $(m+n)$ to implement the simplex method. The following analysis and results are directed 
towards developing a technique to embed GD in the simplex method with a basis of size $\mathrm{m}$. Suppose the weights for a singleton basic variable $x_{k}$ are given by $w_{j k}=\bar{c}_{j} /\left|\bar{c}_{j}\right|= \pm 1\left(\left|\bar{c}_{j}\right|>0\right)$ if $\mathrm{j}=\mathrm{k}$ and zero, otherwise. Now consider any basis of formulation $\operatorname{LP}(3)$ with weights for the $\mathrm{m}$ basic variables $(\mathrm{k}(1), \ldots, \mathrm{k}(\mathrm{m}))$ given by $w_{j k_{(i)}}$, for $\mathrm{i}=(1, \ldots, \mathrm{m})$ and $\mathrm{j}=(1, \ldots$, $\mathrm{n})$. It is clear that the values of the variables $x_{j}$, for $\mathrm{j}=(1,2 \ldots, \mathrm{n})$, in the formulation $\mathrm{LP}(1)$ can be obtained from

$$
\bar{x}_{j}=\sum_{i=1}^{m} w_{j k_{(i)}} \bar{b}_{i} .
$$

Assuming that the selected set of basic directions and the corresponding step lengths yielded all $\bar{x}_{j} \geq 0$ in (5) at some iteration (t-1), consider a new direction $w_{j t}=\bar{w}_{j t} / \gamma$ at iteration $\mathrm{t}$ where

$$
\bar{w}_{j t}= \begin{cases}\bar{c}_{j} & \text { if } \bar{c}_{j}>0 \text { or } \bar{c}_{j}<0 \text { and } \bar{x}_{j}>0 \\ 0 & \text { otherwise }\end{cases}
$$

where $\gamma=\sum_{j=1}^{n}\left|\bar{w}_{j t}\right|$. If there is no such direction clearly the current tableau is optimal. If the step length of the new direction is $\alpha$, then the new solution $\bar{x}_{j}$ is given by

$$
\begin{aligned}
& \bar{x}_{j}=\quad \sum_{i=1}^{m} w_{j k_{(i)}}\left(\bar{b}_{i}-\alpha d_{i t}\right)+\alpha w_{j t} \\
& =\sum_{i=1}^{m} w_{j k_{(i)}} \bar{b}_{i}+\alpha\left(w_{j t}-\sum_{i=1}^{m} w_{j k_{(i)}} d_{i t}\right) \\
& =\quad \bar{x}_{j}+\alpha \theta_{j t}
\end{aligned}
$$

where $d_{i t}=\sum_{j=1}^{n} \bar{a}_{i j} w_{j t}$ and $f_{t}=\sum_{j=1}^{n} \bar{c}_{j} w_{j t}$. If all $\theta_{j t} \geq 0$, the objective function can be made as large as possible by selecting large values for $\alpha$ and hence the problem is unbounded. If for some $\mathbf{j}, \theta_{j t}<0$, then the maximum step length $\alpha_{t}^{*}$ to maintain primal feasibility is given by

$$
\alpha_{t}^{*}=\min _{j}\left(-\bar{x}_{j} / \theta_{j t}, \theta_{j t}<0\right)=-\bar{x}_{r} / \theta_{r t} .
$$

Having determined the step length $\alpha_{t}^{*}$, the following analysis provides a mechanism to pivot a new direction into the basis. Suppose for each variable j, $P(j)=\left(i, w_{j k(i)} \neq 0\right)$ and $\mathrm{NP}(\mathrm{j})$ represents the number of elements in $\mathrm{P}(\mathrm{j})$. It is easy to verify from (7), moving along the generated direction by the step length in (8) reduces the value of $x_{r}$ to zero. There are three cases.

CASE 1: $x_{r}$ is basic and $\mathrm{NP}(\mathrm{r})=1$

Since $x_{r}$ is a basic variable and $\mathrm{NP}(\mathrm{r})=1, \bar{c}_{r}=w_{r t}=0, w_{r k_{(i)}}=0$ when 
$i \neq r$ and $w_{r k(r)}=1$. Therefore from (7), it follows that $\theta_{r t}=-d_{r t}$ which implies $\bar{b}_{k(r)} / d_{r t}=-\bar{x}_{r} / \theta_{r t}=\alpha_{t}^{*}$ and hence the basic variable $x_{r}$ can be replaced by the new direction. If any of the updated $\bar{b}_{i}$ are negative, then change it to $-\bar{b}_{i}$ and multiply the corresponding weights of the direction by -1 .

CASE 2: $\alpha_{t}^{*}>0$ and $x_{r}$ is nonbasic or $\mathrm{NP}(\mathrm{r}) \geq 2$

In this case it is possible to generate more than one direction. Suppose for $\mathrm{v}=1, w_{t}^{v}=\left(w_{j t}^{v}\right), d_{t}^{v}=\left(d_{i t}^{v}\right), f_{t}^{v}>0$ and $\alpha_{t v}^{*}$ are the normalized weights, the elements of the column vector, the coefficient in the objective function and the step length of the first direction. Also let $\bar{x}_{j}^{(v-1)}$ be the values of the variables prior to generating the direction number v. After generating the direction number $\mathrm{v}$, update the values of the variables $x_{j}$ to $\bar{x}_{j}^{v}=$ $\bar{x}_{j}^{(v-1)}+\alpha_{t v}^{*} w_{j t}^{v}$. Using these updated values of $x_{j}$ and the relationship (6), determine the weights of the new direction. If such a direction does not exist or the corresponding $\alpha_{t v}^{*}=0$, or it has been generated before (to prevent zigzagging), stop generating additional directions. Suppose $h$ is the number of directions generated and let

$$
\begin{array}{cc}
\bar{w}_{j t}=\sum_{v=1}^{h}\left(\alpha_{t v}^{*} w_{j t}^{v}\right), & \bar{d}_{i t}=\sum_{v=1}^{h}\left(\alpha_{t v}^{*} d_{i t}^{v}\right) \\
\bar{f}_{t}=\sum_{v=1}^{h}\left(\alpha_{t v}^{*} f_{t}^{v}\right), \quad \lambda=\sum_{j=1}^{n}\left|\bar{w}_{j t}\right| .
\end{array}
$$

The following results are useful in combining these $\mathrm{h}$ directions into a single direction.

LEMma $1 \lambda>0$.

Proof: From (9), $\lambda=0$ implies $\left|\bar{w}_{j t}\right|=\bar{w}_{j t}=\sum_{v=1}^{h} \alpha_{t v}^{*} w_{j t}^{v}=0$ for all j. After generating the $\mathrm{h}$ directions the values of the variables $x_{j}=x_{j}^{h}$ are given by

$$
\bar{x}_{j}^{h}=\bar{x}_{j}^{0}+\sum_{v=1}^{h} \alpha_{t v}^{*} w_{j t}^{v}=\bar{x}_{j}^{0}+\bar{w}_{j t}=\bar{x}_{j}^{0} .
$$

From (10) it follows that the increase in the objective function is zero which is a contradiction since the increase in the objective function is $\bar{f}_{t}>0$.

THEOREM 1 After normalization, let $w_{j t}=\left(\bar{w}_{j t} / \lambda\right), d_{i t}=\bar{d}_{i t} / \lambda$ and $f_{t}=$ $\bar{f}_{t} / \lambda$. Then the step length of the combined direction is $\lambda$. 
Proof: First note that $\bar{c}_{j}$ values remain the same for all the $\mathrm{h}$ directions generated as well as for the combined direction. Also moving a step length of $\alpha_{t v}^{*}$ along each direction for $\mathrm{v}=(1, \ldots, \mathrm{h})$ and the maximum step length $\alpha_{t}^{*}$ along the combined single direction results in the same increase in the objective function. It is clear that the increase in the objective function moving along the combined direction is given by $\alpha_{t}^{*} f_{t}=\alpha_{t}^{*} \sum_{j=1}^{n} \bar{c}_{j} w_{j t}$. Substituting for $w_{j t}$ from (9), it is easy to verify that $\alpha_{t}^{*}=\lambda$.

Having combined all $\mathrm{h}$ directions to a single direction, let $x_{r}$ be a variable driven to zero. If it is a basic variable and $\mathrm{NP}(\mathrm{r})=1$, replace this variable with the direction generated as in the Case 1. Otherwise generate an entering direction by combining the new direction with all basic directions in $\mathrm{P}(\mathrm{r})$. Moving a step length of $\lambda$ along the generated single direction reduces the step lengths of the basic directions (RHS of the simplex tableau) to $\left(\bar{b}_{i}-\lambda d_{i t}\right)$. Using these step lengths as weights, the weights of the entering direction are

$$
\bar{w}_{j}=\sum_{v \in P(r)}\left(\bar{b}_{v}-\lambda d_{v t}\right) w_{j k(v)}+\lambda w_{j t} .
$$

Lemma 2 Suppose $\gamma=\sum_{j=1}^{n}\left|\bar{w}_{j}\right|$. Then $\gamma=0$ implies $\bar{b}_{i}=0$ for all $i \in P(r), d_{i t}=0$ for all $i \notin P(r)$ and $f_{t}=0$.

Proof: Clearly $\gamma=0$ implies $\left|\bar{w}_{j}\right|=\bar{w}_{j}=0$ which in turn follows from (11)

$$
\lambda w_{j t}=\sum_{v \in P(r)} w_{j k(v)}\left(\lambda d_{v t}-\bar{b}_{v}\right)
$$

Multiplying both sides of the equation (12) by $\bar{a}_{i j}$ and summing from $\mathrm{j}=1$ to $\mathrm{n}$ results in (after rearranging the terms)

$$
\lambda \sum_{j=1}^{n} w_{j t} \bar{a}_{i j}=\lambda d_{i t}=\sum_{v \in P(r)}\left(\lambda d_{v t}-\bar{b}_{v}\right) d_{i k(v)}
$$

where $d_{i k(v)}$ are the elements of the column vector of the basic variable $\mathrm{k}(\mathrm{v})$ which is an identity column. Therefore it follows $\lambda d_{i t}=\left(\lambda d_{i t}-\bar{b}_{i}\right)$ for $i \in P(r)$ and $\lambda d_{i t}=0$ for $i \notin P(r)$. To prove the second part multiplying both sides of the equation (12) by $\bar{c}_{j}$ and summing over $\mathrm{j}=1$ to $\mathrm{n}$ results in

$$
\lambda \sum_{j=1}^{n} w_{j t} \bar{c}_{j}=\sum_{v \in P(r)}\left(\lambda d_{v t}-\bar{b}_{v}\right) f_{k(v)}=\lambda f_{t} .
$$


Since $\mathrm{k}(\mathrm{v})$ for $v \in P(r)$ are basic variables, $f_{k(v)}=0$ and therefore $f_{t}=0$.

Corollary 1. When $\lambda>0$ and $f_{t}>0$ then $\gamma>0$.

TheOREm 2 Suppose $\lambda>0$ and $f_{t}>0$. When the weights of the entering direction from(11) are normalized, let $w_{j}=\bar{w}_{j} / \gamma$. Then the column vector $d=\left(d_{i}\right)$ of the entering direction is given by $d_{i}=\bar{b}_{i} / \gamma$ for $i \in P(r)$ and $\lambda d_{i t} / \gamma$ for $i \notin P(r)$.

Proof: By definition the elements of the vector $\mathrm{d}$ are given by $d_{i}=$ $\sum_{j=1}^{n}\left(\bar{a}_{i j} \bar{w}_{j} / \gamma\right)$. Substituting for $\bar{w}_{j}$ from (11) and simplifying yields ( see lemma 2 )

$$
d_{i}=(1 / \gamma) \sum_{v \in P(r)}\left(\bar{b}_{v}-\lambda d_{v t}\right) d_{i k(v)}+(\lambda / \gamma) d_{i t}
$$

where $d_{i k(v)}$ are the elements of the column vector of a basic variable in the current tableau which is an identity column. Therefore $d_{i k(v)}=1$ if $\mathrm{i}=$ $\mathrm{v}$ and zero, otherwise. Substituting for $d_{i k(v)}$ in the above expression the required result follows.

Theorem 3 The step length of the entering direction with weights $w_{j}=$ $\bar{w}_{j} / \gamma$ as in (11) is $\gamma$. In addition, the step lengths of the combined direction and the entering direction yield the same increase in the objective function.

Proof: To determine the step length of the entering direction, let $\theta_{j}$ and $\theta_{j t}$ be the values of $\theta$ corresponding to the entering and combined directions respectively. Then from $(7), \theta_{j}=\left(\bar{w}_{j} / \gamma-\sum_{i=1}^{m} w_{j k(i)} d_{i}\right)$. Substituting for $\bar{w}_{j}$ from (11) and for $d_{i}$ from Theorem(2) results in

$$
\begin{aligned}
\theta_{j}= & (1 / \gamma) \sum_{v \in P(r)}\left(\bar{b}_{v}-\lambda d_{v t}\right) w_{j k(v)}+(\lambda / \gamma) w_{j t} \\
& -\sum_{v \in P(r)} w_{j k(v)}\left(\bar{b}_{v} / \gamma\right)-\sum_{v \notin P(r)} w_{j k(v)}(\lambda / \gamma) d_{i t} \\
= & (\lambda / \gamma)\left[w_{j t}-\sum_{i=1}^{m} w_{j k(i)} d_{i t}\right]=(\lambda / \gamma) \theta_{j t} .
\end{aligned}
$$

Since $\lambda$ is the maximum step length possible along the combined direction and the variable $x_{r}$ is driven to zero at the maximum step length, it follows from (8)

$$
\lambda=\min _{j}\left(-\bar{x}_{j} / \theta_{j t}, \theta_{j t}<0\right)=-\bar{x}_{r} / \theta_{r t} .
$$

Since $\theta_{j}=(\lambda / \gamma) \theta_{j t}$ for all $j=(1, \ldots, n)$, the variable $x_{r}$ is also forced to zero at the maximum step along the entering direction and therefore the 
maximum step length is given by $-x_{r} / \theta_{r}=\left(-x_{r} / \theta_{j t}\right)(\gamma / \lambda)=\gamma$.

Substituting for $\bar{w}_{j}$ from (11) it is straight forward to show that $\mathrm{f}$, the coefficient of entering direction in the objective function is given by

$$
\left.f=\sum_{j=1}^{n} \bar{c}_{j} w_{j}=1 / \gamma\right) \sum_{j=1}^{n} \bar{c}_{j} \bar{w}_{j}=(\lambda / \gamma) f_{t} .
$$

This proves the second part of the result $\gamma f=\lambda f_{t}$.

LEMMA 3 The entering direction can be pivoted into the basis by replacing any of the basic directions $v \in P(r)$ for which $\bar{b}_{v}>0$.

Proof: Note that at least one $\bar{b}_{v}$ for $v \in P(r)$ must be $>0$. Otherwise from (5) it follows that $x_{r}=0$ which is impossible. For $v \in P(r)$ and $\bar{b}_{v} \neq 0, \bar{b}_{v} / d_{v}=\gamma$ and therefor any one of the basic variables $v \in P(r)$ for which $\bar{b}_{v} \neq 0$ can be replaced by the entering column.

Corollary 2. Pivoting the entering direction results in a tableau with $\bar{b}_{i}=0$ for $i \in P(r)$ except for the departing direction which is $\gamma$. In addition the weight of the variable $r$ (the variable driven to zero) in the entering direction is zero.

CASE $3: \alpha_{t}^{*}=0$ and $x_{r}$ is nonbasic or $\mathrm{NP}(\mathrm{r}) \geq 2$

In this case the first direction generated results in $\alpha_{t}^{*}=0$. From (5) and (7), it follows that there exists a variable $r \in v=(1, \ldots, n)$ such that $\bar{x}_{r}$ $=\sum_{i=1}^{m} w_{r k(i)} \bar{b}_{i}=0$ and $\theta_{r}=w_{r t}-\sum_{i=1}^{m} w_{r k(i)} d_{i t}<0$. Since $\bar{x}_{r}=0$ and $w_{r t} \geq 0$ it follows that

$$
\sum_{i=1}^{m} w_{r k(i)} d_{i t}>w_{r t} \geq 0
$$

Suppose $T(r) \subseteq P(r)$ and is given by $\mathrm{T}(\mathrm{r})=\left(i \mid i \in P(r)\right.$ and $\left.\bar{b}_{i}>0\right)$. Clearly $P(r) \neq \emptyset$, otherwise all $w_{r k(i)}=0$ which violates the relationship (13). If $\mathrm{NP}(\mathrm{r})=1$, then there exists a unique $\mathrm{p}$ for which $w_{r k(p)} \neq 0$. From (13), it follows that $d_{p t} \neq 0$. Since $\bar{x}_{r}=0$ implies $\bar{b}_{p}=0$ and therefore the basic variable $\mathrm{k}(\mathrm{p})$ can be replaced by the new direction at zero level. When $N P(r) \geq 2$, suppose $(P(r)-T(r)) \neq \emptyset$ and at least one $d_{i t} \in(P(r)-T(r)) \neq 0$. In this case any basic variable $\mathrm{k}(\mathrm{p})$ for which $d_{p t} \neq 0$ and $p \in(P(r)-T(r))$ can be replaced by the new direction at zero level since $\bar{b}_{p}=0$ for $p \in(P(r)-T(r))$. The following results are directed towards developing a mechanism to pivot the new direction at zero level when $(P(r)-T(r))=\emptyset$ or $(P(r)-T(r)) \neq \emptyset$ and $d_{i t}=0$ for all $i \in(P(r)-T(r))$. 
Lemma 4 If $T(r)=\emptyset$, then there exists a basic variable $k(p)$, for which $\bar{b}_{p}=0$ and $d_{p t} \neq 0$.

Proof: If $T(r)=\emptyset$, then $\bar{b}_{i}=0$ for all $i \in P(r)$. From (13), it follows that there exists at least one $\mathrm{p}$ for which $d_{p t} \neq 0$. Any basic variable $\mathrm{k}(\mathrm{p})$ for which $p \in P(r)$ and $d_{p t} \neq 0$ can be replaced by the new direction.

Lemma 5 If $T(r) \neq \emptyset$, then there are at least two elements in $T(r)(N T(r) \geq$ 2).

Proof: Suppose if possible $\mathrm{NT}(\mathrm{r})=1$. Then there exists a unique $p \in$ $P(r)$ for which $\bar{b}_{p} \neq 0$. It follows that $\bar{x}_{r}=\sum_{i=1}^{m} w_{r k(i)} \bar{b}_{i}=w_{r k(p)} \bar{b}_{p} \neq 0$, which is a contradiction.

Combine all the directions $(N T(r) \geq 2)$ in $\mathrm{T}(\mathrm{r})$ into a single direction with weights $\bar{b}_{i}$ and pivoting this combined direction into the basis by replacing any one of the basic directions in $\mathrm{T}(\mathrm{r})$ provides space for the new direction to be pivoted at zero level. The weights of this combined direction are given by $\bar{w}_{j}=\sum_{i \in T(r)} w_{j k(i)} \bar{b}_{i}$. The following results are useful in determining the column vector of the combined direction.

THEOREM 4 The column vector $\bar{d}=\left(\bar{d}_{i}\right)$ of the combined direction with weights $\bar{w}_{j}$ is given by $\bar{d}_{i}=\bar{b}_{i}$ if $i \in T(r)$ and $\bar{d}_{i}=0 i \notin T(r)$.

Proof: The elements of the vector $\bar{d}$ are given by

$$
\begin{aligned}
\bar{d}_{i} & =\sum_{j=1}^{n} \bar{a}_{i j} \bar{w}_{j}=\sum_{j=1}^{n} \bar{a}_{i j} \sum_{v \in T(r)} \bar{b}_{v} w_{j k(v)} \\
& =\sum_{v \in T(r)} \bar{b}_{v} \sum_{j=1}^{n} \bar{a}_{i j} w_{j k(v)}=\sum_{v \in T(r)} \bar{b}_{v} d_{i k(v)} .
\end{aligned}
$$

Noting the fact that $d_{i k(v)}$ are the elements of the column vector of the basic variable $\mathrm{k}(\mathrm{v})$ which is an identity column vector and $d_{i k(v)}=1$ for i $=\mathrm{v}$ and $d_{i k(v)}=0$ for $i \neq v$, the required result $\bar{d}_{i}=\bar{b}_{i}$ for $i \in T(r)$, and $\bar{d}_{i}=0$ for $i \notin T(r)$ follows.

Lemma 6 Let $\gamma_{1}=\sum_{j=1}^{n}\left|\bar{w}_{j}\right|$. Then $\gamma_{1}>0$.

Proof: Suppose if possible $\gamma_{1}=0$. Then it follows $\bar{w}_{j}=0$ for all $j=$ $(1, \ldots, n)$. Now the elements of the column vector $\bar{d}$ are given by $\bar{d}_{i}=$ $\sum_{j=1}^{n} \bar{a}_{i j} \bar{w}_{j}=0$ for all i including $i \in T(r)$ which contradicts Theorem(4).

It is straight forward to verify by normalizing the weights of the combined direction to $w_{j}=\bar{w}_{j} / \gamma_{1}$, the corresponding elements of the column vector 
are given by $\bar{d}_{i}=\bar{b}_{i} / \gamma_{1}$ for $i \in T(r)$ and $\bar{d}_{i}=0$ for $\notin T(r)$. Now the combined direction can be pivoted by replacing any basic direction $p \in$ $T(r)$. Pivoting this combined direction changes the RHS of the tableau to

$$
\begin{aligned}
\overline{\bar{b}}_{i} & =\bar{b}_{i} \text { for } i \notin T(r) \\
& =\gamma_{1} \text { for } i=p \\
& =0 \text { for } i \in(T(r)-p) .
\end{aligned}
$$

Pivoting the combined direction also changes the column vector of the new direction to

$$
\begin{aligned}
\bar{d}_{i t} & =d_{i t} \text { for } i \notin T(r) \\
& \left.=d_{p t}\left(\gamma_{1}\right) / \bar{b}_{p}\right) \text { for } i=p \\
& =d_{i t}-d_{p t}\left(\bar{b}_{i} / \bar{b}_{p}\right) \text { for } i \notin(T(r)-p) .
\end{aligned}
$$

TheOREM 5 There exists an $i \in(T(r)-p)$ for which $\bar{d}_{i t} \neq 0$.

\section{Proof:}

Since $N T(r) \geq 2,(\mathrm{~T}(\mathrm{r})-\mathrm{p})$ is not empty. Suppose if possible $\bar{d}_{i t}=0$ for all $i \in(T(r)-p)$. Since $\bar{b}_{i}=0$ for $i \in(P(r)-T(r)), w_{r k(i)}=0$ for $i \notin P(r)$ and $\bar{x}_{r}=0$, it follows that $\sum_{i=1}^{m} w_{r k(i)} \bar{b}_{i}=\sum_{i \in T(r)} w_{r k(i)} \bar{b}_{i}=0$. From (14), $\bar{d}_{i t}=0$ implies $d_{i t} \bar{b}_{p}-d_{p t} \bar{b}_{i}=0$ for all $i \in(T(r)-p)$. Substituting for $d_{p t} \bar{b}_{i}$ yields

$$
\begin{aligned}
d_{p t} \sum_{i \in T(r)} w_{r k(i)} \bar{b}_{i} & =\sum_{i \in T(r)} w_{r k(i)} d_{p t} \bar{b}_{i} \\
& =\sum_{i \in(T(r)-p)} w_{r k(i)} d_{i t} \bar{b}_{p}+w_{r k(p)} d_{p t} \bar{b}_{p} \\
& =\bar{b}_{p} \sum_{i \in T(r)} w_{r k(i)} d_{i t}=0
\end{aligned}
$$

Since $d_{i t}=0$ for $i \in(P(r)-T(r))$ and $w_{r k(i)}=0$ for $i \notin P(r)$, it follows that $\bar{b}_{p} \sum_{i=1}^{m} w_{r k(i)} d_{i t}=0$. Since $\bar{b}_{p}>0$, this contradicts (13).

After pivoting the combined direction, the new direction can be pivoted by replacing any basic variable $i \in(T(r)-p)$ for which $\bar{d}_{i t} \neq 0$. Based on the above analysis and results, the following is an implementation of imbedding the full GD in the simplex method. 


\section{Algorithm}

Step(1): Start with an initial set of basic directions. Slack and artificial variables may be used to get started. For $i=(1, \ldots, m)$ and $j=(1, \ldots, n)$, let $w_{j k(i)}$ be the weights of the basic variables, $\bar{A}=\left(\bar{a}_{i j}\right), \bar{b}=\left(\bar{b}_{i}\right)$ and $\bar{c}$ $=\left(\bar{c}_{j}\right)$ be the elements of the initial simplex tableau and $\bar{x}_{j}=\sum_{i=1}^{m} w_{j k(i)} \bar{b}_{i}$.

$\operatorname{Step}(2)$ : Determine the weights of the entering direction $w_{j t}$ from (6) If all $w_{j t}=0$, stop. The current solution is optimal. Otherwise attempt to find the maximum step length $\alpha_{t}^{*}$ of the direction from (7) and (8). If the step length cannot be found stop. The problem is unbounded. Otherwise let $\bar{x}_{r}$ be one of the variables limiting the step length to $\alpha_{t}^{*}$.

Step(3): Let $P(r)=\left(i, w_{r k(i)} \neq 0\right)$ and $\mathrm{NP}(\mathrm{r})$ represents the number of elements in $\mathrm{P}(\mathrm{r})$. If $x_{r}$ is a basic variable and $\mathrm{NP}(\mathrm{r})=1$, go to $\operatorname{Step}(4)$. Otherwise go to $\operatorname{Step}(5)$.

Step(4): Pivot the new direction replacing the basic direction corresponding to the variable $x_{r}$, update the values of the variables and go to $\operatorname{Step}(2)$.

Step(5): In this case $x_{r}$ is either nonbasic or $N P(r) \geq 2$. If $\alpha_{t}^{*}=0$, then go to $\operatorname{Step}(7)$. Otherwise $\left(\alpha_{t}^{*}>0\right)$, update the values of the variables $x_{j}$ and continue generating more directions until such a direction does not exist, or it has been generated before in the current iteration (to prevent zigzagging) or the corresponding step length is zero. If the corresponding step length does not exist stop. The problem is unbounded. Otherwise combine all the directions generated into a single direction by multiplying the weights with step lengths and determine the weights $w_{j t}$ and the elements of the column vector $d_{i t}$ of this single direction from (9) and Theorem(1). Also determine the step length of this single direction $\lambda$ and the limiting variable $x_{r}$. If $x_{r}$ is basic and $N P(r)=1$, go to step(4). Otherwise go to $\operatorname{step}(6)$.

Step(6): Combine all the directions in $\mathrm{P}(\mathrm{r})$, with weights $\left(\bar{b}_{i}-\lambda d_{i t}\right)$ and the single direction with weight $\lambda$. Determine the weights of the entering direction $w_{j}$ and the step length $\gamma$ from (11) and Lemma (2). Also determine the elements of the column vector of the entering direction from Theorem(2). Pivot this entering direction with step length $\gamma$ replacing any of the basic directions in $\mathrm{T}(\mathrm{r})=\left(i \mid i \in P(r)\right.$ and $\left.\bar{b}_{i}>0\right)$. Update the values 
of the variables and go to $\operatorname{step}(2)$.

Step(7): If there exists a basic direction $\mathrm{k}(\mathrm{p})$ for which $\bar{b}_{p}=0$ and the corresponding element of the column vector of the new direction $d_{p t} \neq 0$, then pivot the new direction at zero level by replacing the basic direction $\mathrm{k}(\mathrm{p})$. If $d_{i t}=0$, for all $i \in(P(r)-T(r))$, let $N T(r)$ be the number of elements in $T(r)$ and go to $\operatorname{step}(8)$.

Step(8):.Combine all the basic directions in $T(r)$ with weights $\bar{b}_{i}$ into a single direction and pivot this direction replacing any of the basic directions $k(p)$ in $T(r)$. From (14) determine $\bar{d}_{i t}$, the updated values of the elements of the column vector of the new direction and pivot the new direction with zero step length replacing any of the basic directions $i \in(T(r)-p)$ for which $\bar{d}_{i t} \neq 0$ and go to $\operatorname{step}(2)$.

Note that after pivoting a direction into the basis if any $\bar{b}_{i}<0$, it can be made positive by multiplying the weights of the corresponding direction by $(-1)$. In the next section a numerical example is presented to illustrate the computations involved.

\section{GD and an Example}

In the numerical example in Table $1, x_{4}, x_{5}$, and $x_{6}$ are the slack variables. Starting with the three basic directions $(0,0,0,1,0,0),(0,0,0,0,1,0)$ and $(0,0,0,0,0,1)$ the initial simplex tableau is given in Table 1 . Clearly

Table 1. Example 1-Tableau-1.

\begin{tabular}{|c|c|c|c|c|c|c|c|c|c|}
\hline Basis & $x_{1}$ & $x_{2}$ & $x_{3}$ & $x_{4}$ & $x_{5}$ & $x_{6}$ & RHS & $d_{1}$ & Ratio \\
\hline$x_{4}$ & 1 & 1 & 1 & 1 & 0 & 0 & 5 & 1 & 5 \\
\hline$x_{5}$ & 0 & 1 & 0 & 0 & 1 & 0 & 2 & $1 / 2$ & 4 \\
\hline$x_{6}$ & 1 & 2 & 1 & 0 & 0 & 1 & 6 & $3 / 2$ & 4 \\
\hline $\bar{c}$ & 2 & 3 & 1 & 0 & 0 & 0 & 0 & $7 / 3$ & \\
\hline $\bar{x}$ & 0 & 0 & 0 & 5 & 2 & 6 & & & \\
\hline$w_{1}^{1}$ & $2 / 6$ & $3 / 6$ & $1 / 6$ & 0 & 0 & 0 & & & \\
\hline$\theta_{1}^{1}$ & $2 / 6$ & $3 / 6$ & $1 / 6$ & -1 & $-1 / 2$ & $-3 / 2$ & & & \\
\hline$x^{1}$ & $4 / 3$ & 2 & $2 / 3$ & 1 & 0 & 0 & & & \\
\hline
\end{tabular}

$Q_{1}^{1}=(1,2,3),\left(d_{1}^{1}\right)^{t}=(1,1 / 2,1 / 3), f_{1}^{1}=7 / 3$, and $\alpha_{11}^{*}=4$ resulting in the solution $x^{1}$. Since $Q_{1}^{2}=(1,2,3)=Q_{1}^{1}$ and the basic variable $x_{5}$ (NP (5) $=1$ ) is forced to zero by the first direction, the variable $y_{1}=y_{1}^{1}$ is pivoted 
with step length $\alpha^{*}=4$ replacing $x_{5}$ in Table 1 ( $x_{6}$ could also have been replaced) resulting in Table 2 .

Table 2. Example 1 -Tableau-2.

\begin{tabular}{|c|c|c|c|c|c|c|c|c|c|}
\hline Basis & $x_{1}$ & $x_{2}$ & $x_{3}$ & $x_{4}$ & $x_{5}$ & $x_{6}$ & RHS & $d_{2}$ & Ratio \\
\hline$x_{4}$ & 1 & -1 & 1 & 1 & -2 & 0 & 1 & 1 & 1 \\
\hline$y_{1}$ & 0 & 2 & 0 & 0 & 2 & 0 & 4 & $-5 / 7$ & - \\
\hline$x_{6}$ & 1 & -1 & 1 & 0 & -3 & 1 & 0 & 1 & 0 \\
\hline $\bar{c}$ & 2 & $-5 / 3$ & 1 & 0 & $-14 / 3$ & 0 & $28 / 3$ & $5 / 3$ & - \\
\hline $\bar{x}$ & $4 / 3$ & 2 & $2 / 3$ & 1 & 0 & 0 & & & \\
\hline$w_{2}^{1}$ & $6 / 14$ & $-5 / 14$ & $3 / 14$ & 0 & 0 & 0 & & & \\
\hline$\theta_{2}^{1}$ & $2 / 3$ & 0 & $1 / 3$ & -1 & 0 & -1 & & & \\
\hline$x^{1}$ & $4 / 3$ & 2 & $2 / 3$ & 1 & 0 & 0 & & & \\
\hline
\end{tabular}

Since $Q_{2}^{1}=(1,2,3)$, it follows that $\left(d_{2}^{1}\right)^{t}=(1,-5 / 7,1)$ and $f_{2}^{1}=5 / 3$. Clearly $\alpha_{21}^{*}=0$ due to the basic variable $x_{6}$ with $\mathrm{NP}(6)=1$. Replacing $x_{6}$ with $y_{2}$ results in Table 3 .

Table 3. Example 1-Tableau -3.

\begin{tabular}{|c|c|c|c|c|c|c|c|c|c|}
\hline Basis & $x_{1}$ & $x_{2}$ & $x_{3}$ & $x_{4}$ & $x_{5}$ & $x_{6}$ & RHS & $d_{3}$ & Ratio \\
\hline$x_{4}$ & 0 & 0 & 0 & 1 & 1 & -1 & 1 & $3 / 10$ & $10 / 3$ \\
\hline$y_{1}$ & $5 / 7$ & $9 / 7$ & $5 / 7$ & 0 & $-1 / 7$ & $5 / 7$ & 4 & $-4 / 35$ & - \\
\hline$y_{2}$ & 1 & -1 & 1 & 0 & -3 & 1 & 0 & -1 & - \\
\hline $\bar{c}$ & $1 / 3$ & 0 & $-2 / 3$ & 0 & $1 / 3$ & $-5 / 3$ & $28 / 3$ & $7 / 15$ & - \\
\hline $\bar{x}$ & $4 / 3$ & 2 & $2 / 3$ & 1 & 0 & 0 & & & \\
\hline$w_{3}^{1}$ & $1 / 4$ & 0 & $-2 / 4$ & 0 & $1 / 4$ & 0 & & & \\
\hline$\theta_{3}^{1}$ & $3 / 4$ & $-1 / 4$ & $-1 / 4$ & $-1 / 4$ & $1 / 4$ & 0 & & & \\
\hline$x^{1}$ & $10 / 3$ & $4 / 3$ & 0 & $1 / 3$ & $2 / 3$ & 0 & & & \\
\hline$w_{3}^{2}$ & $1 / 2$ & 0 & 0 & 0 & $1 / 2$ & 0 & & & \\
\hline$\theta_{3}^{2}$ & $5 / 6$ & $-1 / 2$ & $1 / 6$ & $-1 / 2$ & $1 / 2$ & 0 & & & \\
\hline$x^{2}$ & $35 / 9$ & 1 & $1 / 9$ & 0 & 1 & 0 & & & \\
\hline
\end{tabular}

From Table $3, Q_{3}^{1}=(1,3,5),\left(d_{3}^{1}\right)^{t}=(1 / 4,-3 / 14,-1), f_{3}^{1}=1 / 2$, and $\alpha_{31}^{*}=8 / 3$ resulting in the solution $x^{1}$. From $x^{1}$ it is clear that $Q_{3}^{2}=(1,5)$ $\neq Q_{3}^{1},\left(d_{3}^{2}\right)^{t}=(1 / 2,2 / 7,-1), f_{3}^{2}=1 / 3$ and $\alpha_{32}^{*}=2 / 3$ resulting in $x^{2}$. Since the next direction yields $Q_{3}^{3}=(1,3,5)=Q_{3}^{1}$, the directions $y_{3}^{1}$ and $y_{3}^{2}$ are combined in to a single direction with weights $8 / 3$ and $2 / 3$ resulting in the direction $y_{3}$ with weights $8 / 3 w_{3}^{1}+2 / 3 w_{3}^{2}$. Normalizing (dividing by $\lambda=$ $10 / 3)$ yields the weights $(3 / 10,0,-4 / 10,0,3 / 10,0)$. The column vector corresponding to this direction $d_{3}=3 / 10\left(8 / 3 d_{3}^{1}+2 / 3 d_{3}^{2}\right)$ is shown in the Table 3 . The step length of this direction is $\lambda=10 / 3$ which forces the 
basic variable $x_{4}$ to zero. Since $\mathrm{NP}(4)=1$, replacing $x_{4}$ with $y_{3}$ results in Table 4.

Table 4. Example 1-Tableau-4.

\begin{tabular}{|c|c|c|c|c|c|c|c|c|c|}
\hline Basis & $x_{1}$ & $x_{2}$ & $x_{3}$ & $x_{4}$ & $x_{5}$ & $x_{6}$ & RHS & $d_{4}$ & Ratio \\
\hline$y_{3}$ & 0 & 0 & 0 & $10 / 3$ & $10 / 3$ & $-10 / 3$ & $10 / 3$ & $10 / 18$ & 6 \\
\hline$y_{1}$ & $5 / 7$ & $9 / 7$ & $5 / 7$ & $8 / 21$ & $5 / 21$ & $7 / 21$ & $92 / 21$ & $92 / 126$ & 6 \\
\hline$y_{2}$ & 1 & -1 & 1 & $10 / 3$ & $1 / 3$ & $-7 / 3$ & $10 / 3$ & $10 / 18$ & 6 \\
\hline $\bar{c}$ & $1 / 3$ & 0 & $-2 / 3$ & $-14 / 9$ & $-11 / 9$ & $-1 / 9$ & $98 / 9$ & $1 / 54$ & - \\
\hline $\bar{x}$ & $35 / 9$ & 1 & $1 / 9$ & 0 & 1 & 0 & & & \\
\hline$w_{4}^{1}$ & $3 / 20$ & 0 & $-6 / 20$ & 0 & $-11 / 20$ & 0 & & & \\
\hline$\theta_{4}^{1}$ & $83 / 90$ & 0 & $-83 / 90$ & 0 & 0 & 0 & & & \\
\hline$x^{1}$ & 4 & 1 & 0 & 0 & 1 & 0 & & & \\
\hline$w_{4}^{2}$ & $3 / 14$ & 0 & 0 & 0 & $-11 / 14$ & 0 & & & \\
\hline$\theta_{4}^{2}$ & $65 / 63$ & 0 & $-13 / 126$ & 0 & 0 & 0 & & & \\
\hline
\end{tabular}

From Table $4, Q_{4}^{1}=(1,3,5),\left(d_{4}^{1}\right)^{t}=(-11 / 6,-5 / 21,-1 / 3), f_{4}^{1}=83 / 90$, and $\alpha_{41}^{*}=10 / 83$ with the corresponding solution $x^{1}$. The next direction yields $Q_{4}^{2}=(1,5),\left(d_{4}^{2}\right)^{t}=(-55 / 21,-5 / 147,-2 / 42), f_{4}^{2}=65 / 63$ and $\alpha_{42}^{*}=0$. Discarding the second direction and noting the fact that the first direction forced the nonbasic variable $x_{3}$ to zero, combine all the basic directions $y_{3}$, $y_{1}$, and $y_{2}$ in which $x_{3}$ has a nonzero weight and the new direction $y_{4}^{1}$ with weights $[10 / 3+11 / 6(10 / 83)],[92 / 21+5 / 21(10 / 83)],[10 / 3+1 / 3(10 / 83)]$ and $10 / 83$ resulting in the entering direction $y_{4}$ with normalized weights $(4 / 6,1 / 6,0,0,1 / 6,0)$ and step length $\gamma=6$. The corresponding column vector $d_{4}$ is shown in Table 4 . Replacing $y_{3}$ with $y_{4}$, results in Table 5 (note that $y_{1}$ or $y_{2}$ could have also been replaced).

Table 5. Example 1-Tableau-5.

\begin{tabular}{|c|c|c|c|c|c|c|c|c|c|}
\hline Basis & $x_{1}$ & $x_{2}$ & $x_{3}$ & $x_{4}$ & $x_{5}$ & $x_{6}$ & RHS & $d_{5}$ & Ratio \\
\hline$y_{4}$ & 0 & 0 & 0 & 6 & 6 & -6 & 6 & $-24 / 5$ & - \\
\hline$y_{1}$ & $5 / 7$ & $9 / 7$ & $5 / 7$ & -4 & $-29 / 7$ & $33 / 7$ & 0 & $121 / 35$ & 0 \\
\hline$y_{2}$ & 1 & -1 & 1 & 0 & -3 & 1 & 0 & $13 / 15$ & 0 \\
\hline $\bar{c}$ & $1 / 3$ & 0 & $-2 / 3$ & $-5 / 3$ & $-4 / 3$ & 0 & 11 & $17 / 15$ & - \\
\hline $\bar{x}$ & 4 & 1 & 0 & 0 & 1 & 0 & & & \\
\hline$w_{5}^{1}$ & $1 / 5$ & 0 & 0 & 0 & $-4 / 5$ & 0 & & & \\
\hline$\theta_{5}^{1}$ & $17 / 15$ & 0 & $-17 / 15$ & 0 & 0 & 0 & & & \\
\hline
\end{tabular}

The next possible direction is $Q_{5}^{1}=(1,5)$ yields $\alpha_{51}^{*}=0$, due to the nonbasic variable $x_{3}$. Noting the fact that $\mathrm{P}(3)=(2,3)$ and $\mathrm{T}(3)=\emptyset$, 
replacing $y_{1}$ with $y_{5}=y_{5}^{1}$, yields Table 6 (note that $y_{2}$ could also have been replaced).

Table 6. Example 1 -Tableau-6.

\begin{tabular}{|c|c|c|c|c|c|c|}
\hline Basis & $x_{1}$ & $x_{2}$ & $x_{3}$ & $x_{4}$ & $x_{5}$ & $x_{6}$ \\
\hline$y_{4}$ & $120 / 121$ & $216 / 121$ & $120 / 121$ & $54 / 121$ & $30 / 121$ & $66 / 121$ \\
\hline$y_{5}$ & $25 / 121$ & $45 / 121$ & $25 / 121$ & $-140 / 121$ & $-145 / 121$ & $165 / 121$ \\
\hline$y_{2}$ & $56 / 121$ & $-238 / 121$ & $56 / 121$ & $364 / 121$ & $14 / 121$ & $-308 / 121$ \\
\hline $\bar{c}$ & $12 / 121$ & $-51 / 121$ & $-109 / 121$ & $-43 / 121$ & $3 / 121$ & $-187 / 121$ \\
\hline $\bar{x}$ & 4 & 1 & 0 & 0 & 1 & 0 \\
\hline$w_{6}^{1}$ & $4 / 22$ & $-17 / 22$ & 0 & 0 & $1 / 22$ & 0 \\
\hline$\theta_{6}^{1}$ & $459 / 11^{3}$ & 0 & $-459 / 11^{3}$ & 0 & 0 & 0 \\
\hline Basis & RHS & $d_{6}$ & Ratio & & & \\
\hline$y_{4}$ & 6 & $-1581 / 11^{3}$ & - & & & \\
\hline$y_{5}$ & 0 & $-405 / 11^{3}$ & - & & & \\
\hline$y_{2}$ & 0 & $2142 / 11^{3}$ & 0 & & & \\
\hline $\bar{c}$ & 11 & $459 / 11^{3}$ & - & & & \\
\hline
\end{tabular}

Clearly $Q_{6}^{1}=(1,2,5)$ results in $\alpha_{61}^{*}=0$ due to the nonbasic variable $x_{3}$. Since $\mathrm{T}(3)=\emptyset$, replacing $y_{2}$ with the direction $y_{6}=y_{6}^{1}$, results in the optimal Table 7.

Table 7. Example 1-Tableau-7.

\begin{tabular}{|l|llllll|l|}
\hline Basis & $x_{1}$ & $x_{2}$ & $x_{3}$ & $x_{4}$ & $x_{5}$ & $x_{6}$ & RHS \\
\hline$y_{4}$ & $4 / 3$ & $1 / 3$ & $4 / 3$ & $8 / 3$ & $1 / 3$ & $4 / 3$ & 6 \\
$y_{5}$ & $5 / 17$ & $0 / 17$ & $5 / 17$ & $-10 / 17$ & $-20 / 17$ & $15 / 17$ & 0 \\
$y_{6}$ & $44 / 153$ & $-187 / 153$ & $44 / 153$ & $286 / 153$ & $11 / 153$ & $-242 / 153$ & 0 \\
\hline $\bar{c}$ & 0 & 0 & -1 & -1 & 0 & -1 & 11 \\
\hline $\bar{x}$ & 4 & 1 & 0 & 0 & 1 & 0 & - \\
\hline
\end{tabular}

\section{Enhancements for the GD Simplex Method}

Pivoting a new direction into the basis may result in forcing a variable $x_{r}$ to zero for which $\mathrm{NP}(\mathrm{r}) \geq 2$ in $\operatorname{step}(6)$. In steps 7 and 8 , the step length of a new direction is restricted to zero with no improvement in the objective function due to a variable at zero level. If $P(r) \neq \emptyset$ for the variable driven to zero, it may be desirable to eliminate this variable from all basic directions (make the weights to zero). If this is not done, one may have to remove each basic direction in $\mathrm{P}(\mathrm{r})$, one at a time resulting in generating 
and pivoting several new directions requiring substantial computational effort. The following analysis is directed at developing efficient procedures and conditions for feasibility of eliminating a variable driven to zero from all basic directions. There are two cases.

\section{CASE 1: New Direction Step Length $\alpha^{*}>0$}

If $x_{r}$ is basic and $\mathrm{NP}(\mathrm{r})=1$, the basic direction is replaced by the new direction with zero weight for $x_{r}$ and therefore the weight of this variable is zero in all basic directions. If $x_{r}$ is nonbasic and $\mathrm{NP}(\mathrm{r})=1$, the new direction is combined with the unique direction in $\mathrm{P}(\mathrm{r})$ generating the entering direction with zero weight for $x_{r}$ to replace the basic direction $\mathrm{k}(\mathrm{r})$ in $\mathrm{P}(\mathrm{r})$ resulting in zero weights for $x_{r}$ in all basic directions ( see Lemma(3) and Corollary(2)). Now suppose that $x_{r}$ is basic and $N P(r) \geq 2$. If the corresponding RHS of this basic variable $\bar{b}_{r} \neq 0$, then the entering direction with zero weight for $x_{r}$ can be pivoted replacing the basic direction $\mathrm{k}(\mathrm{r})$. This makes $x_{r}$ nonbasic and it still has nonzero weights in other basic directions. But when $\bar{b}_{r}=0$, it is not possible to replace the basic direction $\mathrm{k}(\mathrm{r})$. However, since the column corresponding to $x_{r}$ is an identity column and the value of this variable is zero after pivoting, the weights of the variable $x_{r}$ can be made to zero without impacting the identity columns of the other basic variables. The changes needed are to normalize the weights of the other basic directions by replacing $w_{j k(i)}$ with $w_{j k(i)} / \bar{w}_{r k(i)}$ for $i \neq r$ and $j \neq r$ and $w_{r k(i)}=0$ for $i \neq r$ and replace the RHS of the basic directions from $\bar{b}_{i}$ to $\bar{b}_{i} \bar{w}_{r k(i)}$ (note that $\bar{w}_{r k(i)} \neq 0$ ) where $\bar{w}_{r k(i)}=1-\left|w_{r k(i)}\right|$. This reduces the problem to eliminating a nonbasic variable at zero level with $N P(r) \geq 1$ from all basic directions. Making the weights $w_{r k(i)}$ of the nonbasic variable $x_{r}$ to zero changes the identity columns of the basic variable $\mathrm{k}(\mathrm{p})$ for $\mathrm{p}=(1, \ldots, m)$ to

$$
\begin{aligned}
g_{i p} & =-\bar{a}_{i r} w_{r k(p)} / \bar{w}_{r k(p)} \quad \text { for } i \neq p \\
g_{i i} & =\left(1-\bar{a}_{i r} w_{r k(i)}\right) / \bar{w}_{r k(i)} .
\end{aligned}
$$

Also the $\bar{c}$ values of the basic variables are changed from zero to $\bar{c}_{p}=$ $-\bar{c}_{r} w_{r k(p)} / \bar{w}_{r k(p)}$. Making the columns of the basic variables to identity columns is equivalent to multiplying the matrix $\bar{A}=\left(\bar{a}_{i j}\right)$ with the inverse of the matrix $\mathrm{G}=\left(g_{i p}\right)$. In addition the $\bar{c}$ values of the basic variables must be made to zero. Finally the RHS of the tableau is also changed to $\bar{b}_{p} \bar{w}_{r k(p)}$ from $\bar{b}_{p}$. The following results provide the inverse of the matrix 
$\mathrm{G}$ and the condition when it exists and the justification for changing the $\bar{b}_{p}$ and $\bar{c}_{p}$ values of the basic variables.

THEOREM 6 The inverse of the matrix $G, G^{-1}$ exists if $\Delta=1-\sum_{i=1}^{m} \bar{a}_{i r} w_{r k(i)}$ $\neq 0$ and the elements of the matrix $G^{-1}=\left(\bar{g}_{p j}\right)$ are given by

$$
\begin{aligned}
& \bar{g}_{p j}=\bar{a}_{p r} w_{r k(j)} \bar{w}_{r k(p)} / \Delta \text { forp } \neq j \\
& \bar{g}_{j j}=\left(1+\bar{a}_{j r} w_{r k(j)} / \Delta\right) \bar{w}_{r k(j)} .
\end{aligned}
$$

Proof: It is straight forward to verify the result by multiplying the ith row of the matrix $\mathrm{G}$ with the jth column of the matrix $G^{-1}$.

Lemma 7 When the variable $x_{r}$ is removed from all basic directions and the remaining weights are normalized, the $\bar{c}_{p}$ values of the basic variables are changed to $\bar{c}_{p}=-\bar{c}_{r} w_{r k(p)} / \bar{w}_{r k(p)}$.

Proof: Before the variable $x_{r}$ is removed from all basic directions, the $\bar{c}$ values of the basic variables are given by $\bar{c}_{p}=\sum_{j=1}^{n} w_{j k(p)} \bar{c}_{j}=0$. Deleting the weight $w_{r k(p)}$ and normalizing the remaining weights changes the value to $\bar{c}_{p}=\sum_{j \neq r} \bar{c}_{j} w_{j k(p)} / \bar{w}_{r k(p)}=-\bar{c}_{r} w_{r k(p)} / \bar{w}_{r k(p)}$.

LEMMA 8 When the columns of the $x$-variables are multiplied by the matrix $G^{-1}$, the RHS of the tableau is changed to $\bar{b}_{i} \bar{w}_{r k(i)}$.

Proof: The proof is straight forward by multiplying $G^{-1}$ and the column vector of the RHS of the tableau.

To eliminate the weights of the variable $x_{r}$, from all basic directions first multiply the tableau by $G^{-1}$. Then multiply the pth row of the tableau with $-\bar{c}_{p}$ ( see Lemma(7)) and add it to the $\bar{c}$ row for all $\mathrm{p}$ to make the $\bar{c}_{p}$ of the basic variables to zero. Finally change the weights of the basic directions and the RHS to $w_{j k(p)} / \bar{w}_{r k(p)}$ and $\bar{b}_{p} \bar{w}_{r k(p)}$.

\section{CASE 2: New Direction Step Length $\alpha^{*}=0$}

The analysis is similar to the case when the step length $\alpha^{*}>0$ in all situations except when $x_{r}$ is basic, the corresponding $\bar{b}_{k(r)} \neq 0$ and $\mathrm{NP}(\mathrm{r})$ $\geq 2$. In this case combine all basic directions in $\mathrm{T}(\mathrm{r})$ ( note that $\mathrm{T}(\mathrm{r}) \neq \emptyset$ ), with weights $\bar{b}_{i}$ for $i \in T(r)$ as in step (8) and pivot this direction replacing any direction in $\mathrm{T}(\mathrm{r})$, except the basic variable $x_{r}$. This will make $\bar{b}_{k(r)}=0$ in the updated tableau. Now the variable $x_{r}$ can be eliminated from all basic directions except $\mathrm{k}(\mathrm{r})$ as discussed in the case when $\alpha^{*}>0$. Even before pivoting the new direction, it may be possible to remove the variable 
$x_{r}$ which caused the zero step length from all basic directions. This may help to reduce the number of pivots with zero step length.

To illustrate the computations involved consider the example of the previous section. In the first three tableaus a basic variable is forced to zero. But in Table 4 the entering direction with step length 10/83 forced the nonbasic variable $x_{3}$ to zero. After the new direction is combined with all basic directions in $\mathrm{P}(3)=(1,2,3)$ and pivoting this direction resulted in Table 5. The elements of the column vector of $x_{3}$ in Table 5 are $\bar{a}_{13}=0$, $\bar{a}_{23}=5 / 7$, and $\bar{a}_{33}=1$. Also the weights of $x_{3}$, in the three basic directions are $w_{3 k(1)}=0, w_{3 k(2)}=1 / 6$ and $w_{3 k(3)}=3 / 14$. The matrix $\mathrm{G}$ and its inverse $G^{-1}$ are given by

$$
\mathrm{G}=\left|\begin{array}{lll}
1 & 0 & 0 \\
0 & 37 / 35 & -15 / 77 \\
0 & -1 / 5 & 1
\end{array}\right| G^{-1}=\left|\begin{array}{lll}
1 & 0 & 0 \\
0 & 55 / 36 & 75 / 392 \\
0 & 11 / 56 & 407 / 392
\end{array}\right|
$$

Removing the variable $x_{3}$ from all basic directions changes the $\bar{c}$ values of the three basic variables to $\bar{c}_{y_{4}}=0, \bar{c}_{y_{2}}=2 / 15$ and $\bar{c}_{y_{2}}=2 / 11$. Multiplying the columns of the x-variables of Table 5 with $G^{-1}$ and then multiplying the rows of the Table 5 with $0,(-2 / 15)$ and $(-2 / 11)$ and adding them to the $\bar{c}$ row results in the optimal tableau below (Table 8).

Table 8. Example 2 -Optimal Tableau.

\begin{tabular}{|l|lllllll|}
\hline Basis & $x_{1}$ & $x_{2}$ & $x_{3}$ & $x_{4}$ & $x_{5}$ & $x_{6}$ & RHS \\
\hline$y_{4}$ & 0 & 0 & 0 & 6 & 6 & -6 & 6 \\
$y_{1}$ & $25 / 28$ & $30 / 28$ & $25 / 28$ & $-110 / 28$ & $-130 / 28$ & $135 / 28$ & 0 \\
$y_{2}$ & $33 / 28$ & $-22 / 28$ & $33 / 28$ & $-22 / 28$ & $-110 / 28$ & $55 / 28$ & 0 \\
\hline $\bar{c}$ & 0 & 0 & -1 & -1 & 0 & -1 & 11 \\
\hline
\end{tabular}

\section{Expanded Basis Gradient Simplex Method}

In steps (6) of the algorithm a new direction is combined with all basic directions in $\mathrm{P}(\mathrm{r})$ before it can be pivoted into the basis. In step (8), all basic directions in $\mathrm{T}(\mathrm{r})$ are combined into a single direction and it is pivoted to make room to pivot the new direction with zero step length. A possible approach to avoid combining the basic directions or combining with the basic directions is to expand the basis size. As noted earlier, if the variable $x_{r}$ which is forced to zero is a basic variable and $\mathrm{NP}(\mathrm{r})=1$, then the 
direction can be pivoted replacing $x_{r}$. Otherwise consider the relationship $u_{r}-x_{r}-\sum_{i \in P(r)} w_{r k(i)} y_{i}=0$, where $y_{i}$ are the basic directions pivoted into the basis so far. Clearly $u_{r}$ yields the value of the variable $x_{r}$ and is a redundant constraint. Adjoin this constraint at the bottom of the tableau which increases the size of the basis by one. Suppose the current basis is $v \geq m$. Noting the fact that $y_{i}=\bar{b}_{k(i)}-\sum_{j=1}^{n} \bar{a}_{k(i) j} x_{j}$ and substituting for $y_{i}$ in the expression for $u_{r}$ yields the coefficient $\bar{a}_{v+1 j}$ of $x_{j}$ and is given by $\bar{a}_{v+1 j}=\sum_{i \in P(r)} \bar{a}_{i j} w_{r k(i)}$ if $j \neq r$ and $\bar{a}_{v+1 r}=\sum_{i \in P(r)} \bar{a}_{i r} w_{r k(i)}-1$.

TheOREM 7 The element of the column vector of the entering direction corresponding to the new constraint $d_{v+1 t}=-\theta_{r}$.

Proof: By definition

$$
\begin{aligned}
d_{v+1 t} & =\sum_{j=1}^{n} w_{j t} \bar{a}_{v+1 j}=\sum_{j=1}^{n} w_{j t} \sum_{i \in P(r)} \bar{a}_{i j} w_{r k(i)}-w_{r t} \\
& =\sum_{i \in P(r)} w_{r k(i)} \sum_{j=1}^{n} \bar{a}_{j t} w_{j t}-w_{r t}=\sum_{i \in P(r)} w_{r k(i)} d_{i t}-w_{r t} \\
& =-\theta_{r} .
\end{aligned}
$$

This proves the required result.

Since the RHS of the equation for $u_{r}$ is $\sum_{i \in P(r)} \bar{b}_{i} w_{r k(i)}=\bar{x}_{r}$, the variable $u_{r}$ can be replaced by the new direction with step length $\alpha^{*}$. The variable $u_{r}$ is discarded after pivoting the new direction. The computations involved are illustrated using the example of section(6). Since the basic variables $x_{5}, x_{6}$ and $x_{4}$ are forced to zero in the first three iterations, the first four tableaus generated under both the methods are identical. In Table 4 since a nonbasic variable $x_{3}$ is forced to zero, the basis size is expanded form 3 to 4 to include the constraint $u_{3}-x_{3}-1 / 6 y_{1}-3 / 14 y_{2}+4 / 10 y_{3}=0$, where $1 / 6$, $3 / 14$ and $-4 / 10$ are the weights of the variable $x_{3}$ in the basic directions $y_{1}$, $y_{2}$ and $y_{3}$. Now substituting for the basic directions $y_{1}, y_{2}$ and $y_{3}$ which are given by

$$
\begin{aligned}
& y_{1}=92 / 21-5 / 7 x_{1}-9 / 7 x_{2}-5 / 7 x_{3}-8 / 21 x_{4}-5 / 21 x_{5}-7 / 21 x_{6} \\
& y_{2}=10 / 23-x_{1}+x 2-x 3-10 / 3 x_{4}-1 / 3 x_{5}+7 / 3 x_{6} \\
& y_{3}=10 / 3-10 / 3 x_{4}-10 / 3 x_{5}+10 / 3 x_{6}
\end{aligned}
$$

from Table 4 , results in $u_{3}+1 / 3 x_{1}-2 / 3 x_{3}-5 / 9 x_{4}-11 / 9 x_{5}+8 / 9 x_{6}=1 / 9$ and the following Table 9 .

Pivoting $y_{4}$ and replacing $u_{3}$ yields the optimal tableau with $\bar{c}=(0,0,0$,$1,0,-1), y_{1}=366 / 83, y_{2}=280 / 83, y_{3}=295 / 83$, and $y_{4}=10 / 83$. It is 
Table 9. Example 3-Tableau-4.

\begin{tabular}{|l|llllll|l|l|}
\hline Basis & $x_{1}$ & $x_{2}$ & $x_{3}$ & $x_{4}$ & $x_{5}$ & $x_{6}$ & RHS & $d_{4}$ \\
\hline$y_{3}$ & 0 & 0 & 0 & $10 / 3$ & $10 / 3$ & $-10 / 3$ & $10 / 3$ & $-11 / 6$ \\
$y_{1}$ & $5 / 7$ & $9 / 7$ & $5 / 7$ & $8 / 21$ & $5 / 21$ & $7 / 21$ & $92 / 21$ & $-5 / 21$ \\
$y_{2}$ & 1 & -1 & 1 & $10 / 3$ & $1 / 3$ & $-7 / 3$ & $10 / 3$ & $-1 / 3$ \\
$u_{3}$ & $1 / 3$ & 0 & $-2 / 3$ & $-5 / 9$ & $-11 / 9$ & $8 / 9$ & $1 / 9$ & $83 / 90$ \\
\hline $\bar{c}$ & $1 / 3$ & 0 & $-2 / 3$ & $-14 / 9$ & $-11 / 9$ & $-1 / 9$ & $98 / 9$ & $83 / 90$ \\
\hline
\end{tabular}

interesting to note that the reduced cost for all variables which are included in the basic directions are all zero which are different when compared with the previous methods.

\section{Remarks and Conclusions}

Imbedding the RGD direction in the frame work of the simplex method is straight forward since a unique direction is generated from each tableau and is pivoted replacing one of the basic direction. Incorporating the GD direction in the simplex method is complicated and differs in three respects. First the step length of each direction is determined by the nonnegativity restrictions on the variables. Second, it is possible to generate many directions with a positive step length from each tableau. These directions must be combined into a single direction. When a basic variable $x_{r}$ is driven to zero and $\mathrm{NP}(\mathrm{r})=1$, the single direction is pivoted replacing the basic variable. Otherwise, the single direction is combined again with all basic direction in $\mathrm{P}(\mathrm{r})$ and pivoted replacing any basic direction in $\mathrm{T}(\mathrm{r})$. Third, when the step length of the first direction is zero, it may be necessary to combine all basic directions in $\mathrm{T}(\mathrm{r})$ ( when $\mathrm{T}(\mathrm{r}) \neq \emptyset$ ) into a single direction and pivot it to make room for pivoting the new direction at zero level. A computationally efficient method is proposed requiring only to multiply the simplex tableau with a matrix, to eliminate a nonbasic variable at zero level under fairly general conditions. This will reduce the number of zero pivots and possibly reduce the number of iterations to determine the optimal solution. In the Expanded Basis Method when a new direction cannot be pivoted replacing a basic direction, a redundant constraint is added to make room to pivot the new direction.

In this paper the results are extended to include a general gradient direction in the frame work of the simplex method. The concept of combining directions is introduced and is used to develop pivoting rules for entering directions. One may limit the number of variables selected in both RGD 
and GD directions without significant changes in the proposed methods. The motivation for limiting the number of variables is that an optimal solution can always be found with no more that $\mathrm{m}$ variables at a positive level. The feasibility of reducing the basis size in the Expanded Basis Method and computational experiments for the two methods presented are under investigation.

\section{Acknowledgments}

The author is grateful to anonymous referees and the area editor for constructive comments on earlier version of this paper and to a colleague Dr. Danielle Fowler for help with the Latex Package.

\section{References}

1. P. Abel. On the Choice of the Pivot Columns of the Simplex Method: Gradient Criteria. Computing,13: 13-21, 1987.

2. K. M. Anstreicher and T. Terlaky. A Monotonic Build-up Simplex Algorithm for Linear Programming. Operations Research, 48: 556-561, 1994.

3. M. H. Beilby. Economics and Operations Research. Academic Press, 25-41, 1976.

4. R. G. Bland. New Finite Pivoting Rules for the Simplex Method. Mathematics of Operations Research, 2: 103-107, 1977.

5. H- D. Chen, P. M.. Pardalos and M.. A. Saunders. The Simplex Algorithm with a New Primal and Dual Pivot Rule. Operations Research Letters, 16: 121-127, 1994.

6. M. C. Cheng. Generalized Theorems for Permanent Basic and Nonbasic Variables. Mathematical Programming, 31: 229-234, 1985.

7. G. B. Dantzig. Linear Programming and Extensions. Princeton University Press, Princeton, New Jersey, 1963.

8. H. A. Eiselt and C- L. Sandblom. Experiments with External Pivoting. Computers and Operations Research, 17: 325-332,1990.

9. Y. Fathi and K. G. Murty. Computational Behavior of a Feasible Direction Method for Linear Programming. European Journal of Operational Research,40: 322-328, 1989.

10. P. E. Gill, W. Murray, M. A. Saunders, and M. A. Wright. A Practical Anti-Cycling Procedure for Linearly Constrained Optimization. Mathematical Programming, 45: 437-474,1989.

11. D. Goldfarb and J. K. Reid. A Practicable Steepest-Edge Simplex Algorithm. Mathematical Programming, 12: 361-371, 1977.

12. R. L. Graves and P. Wolfe. Recent Advances in Mathematical Programming. McGraw-Hill, New York, San Francisco, Toronto, London, 76-77, 1964.

13. P. M. J. Harris. Pivot Selection Methods of the Devex LP Code. Mathematical Programming, 5: 1-28, 1973.

14. E. O. Heady and W. Candler. Linear Programming Methods. The Iowa State College Press, Ames, 560-563, 1958.

15. M. Kallio and E. L. Porteus. A Class of Methods for Linear Programming. Mathematical Programming, 14: 161-169, 1978. 
16. B. Kreko. Linear Programming (Translated by J.H.L. Ahrens and C.M. Safe). Sir Issac Pitman \& Sons, London, 258-271, 1968.

17. G. Mitra, M..Tamiz and J. Yadegar. A Hybrid Algorithm For Linear Programs em Simulation and Optimization of Large Systems, Edited By A. J. Osiadacz, Clarendon Press, Oxford, 1988.

18. S. R. Paranjape. The Simplex Method: Two Basic Variables Replacement. Management Science, 12: 135-141, 1965.

19. J. B. Rosen. The Gradient Projection Method for Nonlinear Programming - Part I: Linear Constraints. Journal of Society of Industrial and Applied Mathematics, 9: 181-217, 1961.

20. T. Terlaky and S. Zhang. Pivot Rules for Linear Programming: A Survey on Recent Theoretical Developments. Annals of Operations Research, 46: 203-233, 1993.

21. Y. Ye. Eliminating Columns in the Simplex Method for Linear Programming. Journal of Optimization Theory and Applications, 63: 69-77, 1989.

22. S. Zhang. On Anti-Cycling Pivoting Rules for the Simplex Method. Operations Research Letters, 10: 189-192, 1992.

23. P. H. Zipkin. Bounds on the Effect of Aggregating Variables in Linear Programs. Operations Research, 28: 403-418, 1980.

24. G. Zoutendijk Mathematical Programming Models. North Holland Publishing Company, Amsterdam, New York, Oxford, 99-115, 1976 


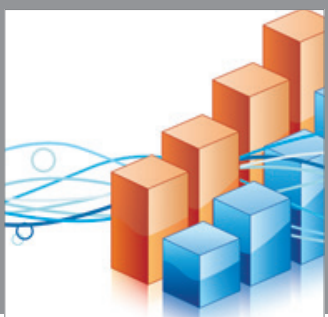

Advances in

Operations Research

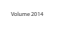

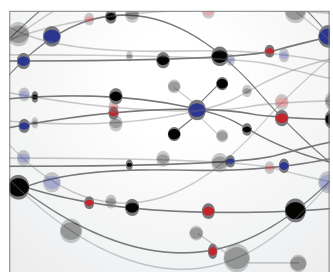

\section{The Scientific} World Journal
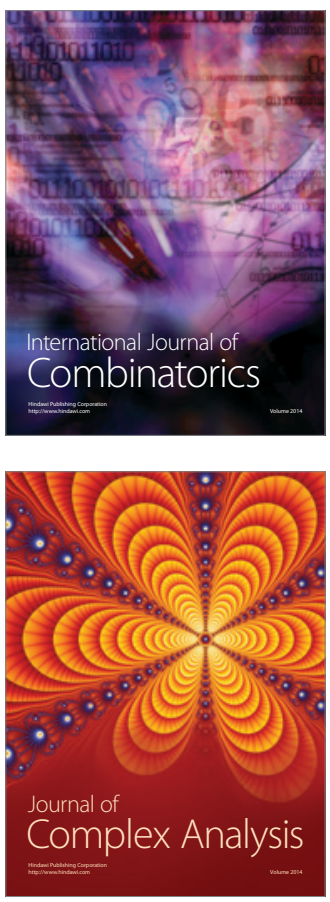

International Journal of

Mathematics and

Mathematical

Sciences
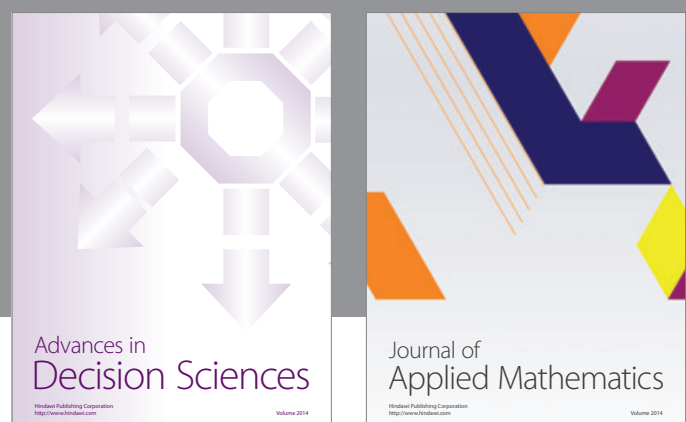

Journal of

Applied Mathematics
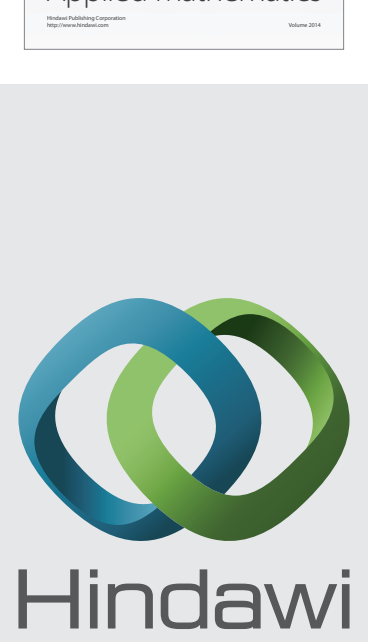

Submit your manuscripts at http://www.hindawi.com
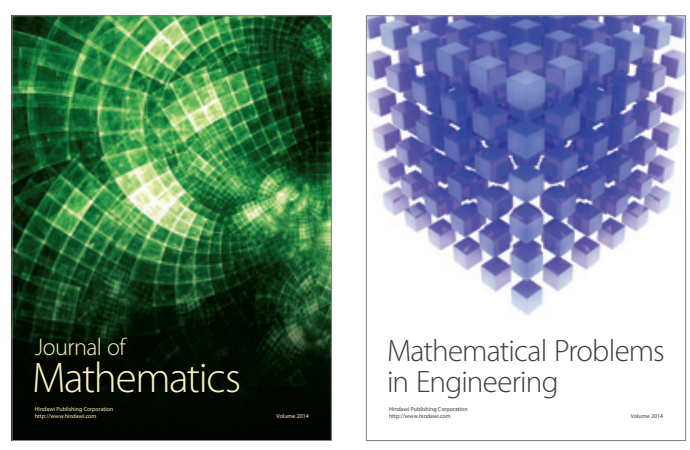

Mathematical Problems in Engineering
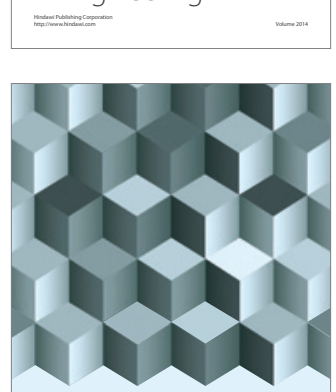

Journal of

Function Spaces
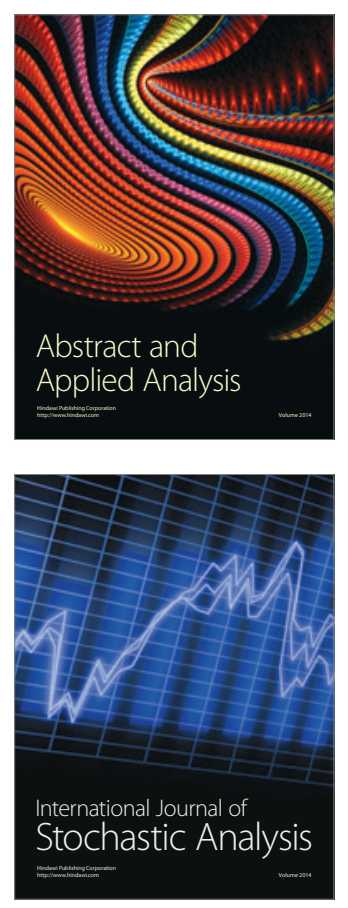

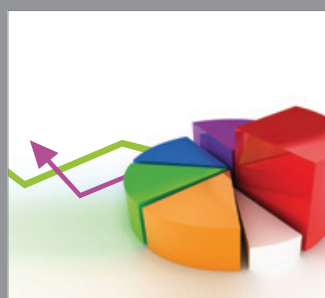

ournal of

Probability and Statistics

Promensencen
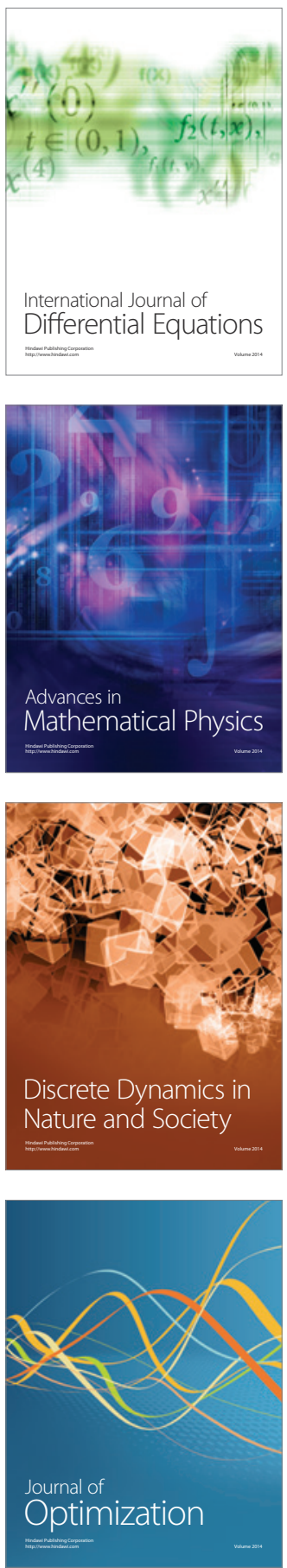